Juntendo Medical Journal

2018. 64 (Suppl 1), 36

\title{
A Deaf Footballer with the Bilateral Intractable Patellar Tendinopathy: a Case Report
}

\author{
Hirofumi NISHIO*1), Yoshitomo SAITA*1), TOMOHIKo TATEISHI*2), \\ YOHEI KOBAYASHI*1), HIROSHI IKEDA*1), KAZUO KANEKO*1) \\ *1) Department of Orthopedic Surgery and Sports Medicine, Juntendo Hospital, Tokyo, Japan, \\ *2) Department of Orthopedics, The Fraternity Memorial Hospital, Tokyo, Japan
}

\begin{abstract}
Background: Deaf football is the football for people with a handicap in hearing. Recently, although the deaf football comes to be spreading in Japan, the medical supports are not enough at all. There are few documents about sports injuries of the deaf football. Therefore there will be a lot of players who play while suffering from any symptoms.

Object: The aim of this case report is to describe the treatment course of a deaf footballer with the bilateral intractable patellar tendinopathy.

Case: The case is a 31 years old man. He is a member of the Japan national team of deaf football. He began deaf football at university student. He continued suffering from a symptom of the patellar tendinopathy since second grade at a high school. He became the first medical examination in our hospital in January 2017. On the first medical examination, he suffered from bilateral patellar tendinopathy. Both sides confirmed severe degenerative changes of the patellar tendon with ultrasonography (Fritschy classification pahse3). We started medical support to him. At first, we introduced physiotherapy as basic treatment to him and his athletic trainer for the improvement of his physical risk factors. With the start of the physiotherapy, we started platelet-rich plasma (PRP) therapy for the purpose of assisting for organization healing process. Both sides gave three times PRP therapy in total every two weeks. Just three months after starting supports, VAS and VISA-P score were improved (VAS: Lt 98 to 48 Rt 88 to 20, VISA-P: Lt 38 to 60 Rt 48 to 80). He participated in DEAFLYMPICS held in Turkey in July 2017.
\end{abstract}

Discussion: The deaf footballers seem to repeat contact injuries because of behind with noticing the existence of the opponents due to their handicaps of hearing. Besides, they are also forced to do more stop motions and cutting movements suddenly. So we think that their injuries are easy to become chronic. Although we were able to experienced good treatment course for a deaf footballer who suffered from the bilateral intractable patellar tendinopathy by the same treatment approaches as physically non-handicapped athletes in this case, there are any problems on medical supports of deaf football.

We propose that:

(1) The epidemiological studies about injuries of deaf football are required.

(2) The deaf footballers are athletes and should be treated like physically non-handicapped athletes.

(3) We should provide more informations of the injury and environments of the care for them in Japan.

For the development of disabled sports, we think that it is essential and important to spread deaf football and provide more medical supports.

Key words: deaf sports, deaf football, intractable patellar tendinopathy, platelet-rich plasma (PRP)

Corresponding author: Hirofumi Nishio

Department of Orthopedic Surgery and Sports Medicine, Juntendo Hospital

2-1-1 Hongo, Bunkyo-ku, Tokyo 113-8421, Japan

TEL: +81-3-3813-3111 E-mail: jmp4ubitj@gmail.com

〔Received Feb. 11, 2018〕〔Accepted Feb. 13, 2018〕

Copyright (C) 2018 The Juntendo Medical Society. This is an open access article distributed under the terms of Creative Commons Attribution License (CC BY), which permits unrestricted use, distribution, and reproduction in any medium, provided the original source is properly credited. doi: $10.14789 /$ jmj. 2018.64.JMJ18-P17 